\title{
Hipermetropia após ceratotomia radial: flutuação da refração e da acuidade visual entre manhã e tarde e correlações com a pressão ocular e o estado biomecânico da córnea
}

\author{
Hyperopia after radial keratotomy: fluctuation of refraction \\ and visual acuity from morning to evening and correlations \\ with intraocular pressure and corneal biomechanics
}

Renato Ambrósio Jr ${ }^{1,2,3}$; Renata Siqueira da Silva ${ }^{1,2}$; Karla Lopes1; Bruno Valbon",2; Allan Luz ${ }^{2,3}$; Guillermo Coca Velarde ${ }^{4}$

\section{Resumo}

\begin{abstract}
Objetivo: Verificar as variações entre manhã e tarde da refração, da acuidade visual, das medidas da pressão ocular e dos parâmetros biomecânicos da córnea em pacientes operados de ceratotomia radial e interesse em retratamento refrativo; correlacionar os parâmetros biomecânicos da córnea com a refração, com a acuidade visual e com as suas variações. Métodos: Foram examinados trinta e oito olhos de 19 pacientes pela manhã $(9 \mathrm{am})$ e à tarde $(6 \mathrm{pm})$, obtendo-se refração esfero-cilíndrica dinâmica, acuidade visual (logMAR) sem correção (AVsc) e corrigida (AVcc) e parâmetros do ORA (Ocular Response Analyzer): histerese corneana (corneal hysteresis - CH), fator de resistência da córnea (corneal resistance factor - CRF), pressão intraocular (intraocular pressure - IOP) calibrada para o padrão Goldmann (IOPg) e pressão compensada da córnea (IOPcc). Considerando a não distribuição normal das variáveis (teste de Kolmogorov-Smirnov), o teste de Wilcoxon signed rank foi utilizado para verificar significância nas diferenças entre as medidas da manhã e as da tarde de cada variável. O teste de Spearman foi utilizado para verificar correlações das medidas do ORA com o estado refracional e com a acuidade visual pela manhã e à tarde, assim como para verificar as correlações entre as medidas do ORA pela manhã e à tarde e as variações da refração e da acuidade visual. Resultados: Grau esférico (E), equivalente esférico (EE), equivalente desfoco (ED), AVsc, IOPcc e IOPg variaram significativamente (Wilcoxon, $\mathrm{p}<0,05)$ entre manhã e tarde, havendo maior hipermetropia, pior acuidade visual não corrigida, maior pressão ocular e menor $\mathrm{CH}$ pela manhã. Nas medidas pela manhã, observaram-se correlações positivas (Spearman, $\mathrm{p}<0,05$ ) do EE e do ED com IOPcc (rs $=0,39$ e 0,34 respectivamente), mas não com IOPg. Nas medidas da tarde, não houve correlações significantes entre os parâmetros refracionais e os pressóricos. Observaram-se correlações negativas (Spearman, $\mathrm{p}<0,05$ ) entre AVsc (logMAR) e CH pela manhã e à tarde $(\mathrm{rs}=-0,48$ e rs= $-0,51)$, entre $\mathrm{E}$ e $\mathrm{CH}$ pela manhã e à tarde ( $\mathrm{rs}=-0,66$ e $-0,76)$, entre $\mathrm{E}$ e $\mathrm{CRF}$ pela manhã e à tarde $(\mathrm{rs}=-0,40$ e -0,47), entre EE e CH pela manhã e à tarde (rs= - 0,70 e -0,68), entre EE e CRF pela manhã e à tarde (rs= $-0,41$ e - $-0,46)$, entre ED e CH pela manhã e a tarde ( $r s=-0,64$ e $-0,54)$ e entre ED e CRF pela manhã e a tarde (rs= - 0,35 e -0,34). Observou-se correlação significante e negativa do CRF pela manhã com a variação do $\mathrm{ED}(\mathrm{p}=0,05 ; \mathrm{rs}=-0,30)$ e com a variação da $\mathrm{AVsc}(\mathrm{p}=0,04 ; \mathrm{rs}=-0,33)$. Conclusão: Maior hipermetropia pela manhã foi associada a maior pressão compensada (IOPcc), mas não com IOPg em pacientes operados de ceratotomia radial, o que deve ser considerado no planejamento do retratamento refrativo. Parâmetros biomecânicos ( $\mathrm{CRF}$ e $\mathrm{CH})$ mais baixos foram associados com maior hipermetropia e pior acuidade visual. Uma tendência de haver maior flutuação relacionada com córneas mais fracas foi encontrada. Novos estudos envolvendo parâmetros biomecânicos derivados do sinal do ORA, além dos parâmetros CH e CRF (derivados das pressões de aplanamento do ORA), juntamente com dados tomográficos da córnea e de aberrometria total são necessários.
\end{abstract} visual

Descritores: Ceratotomia radial/efeitos adversos; Hiperopia/etiologia; Córnea/fisiologia; Erros de refração; Acuidade

${ }^{1}$ Instituto de Olhos Renato Ambrósio

${ }^{2}$ Grupo de Estudos de Tomografia e Biomecânica de Córnea do Rio de Janeiro

${ }^{3}$ Universidade Federal de São Paulo

${ }^{4}$ Universidade Federal Fluminense (Departamento de Estatística)

Os autores declaram inexistir conflitos de interesse

Recebido para publicação em 4/4/2011 - Aceito para publicação em 26/6/2011 


\begin{abstract}
Purpose: To verify the morning to evening variations of refraction, visual acuity, intraocular pressure and biomechanical parameters on patients operated by radial keratotomy who presented for refractive re-treatments; and to correlate the biomechanical parameters with refraction, visual acuity and their variations among morning and evening. Methods: 19 patients were examined, respectively thirty-eight eyes in the morning ( $9 \mathrm{am})$ and evening ( $6 \mathrm{pm})$, recording sphere-cylindrical dynamic refraction, visual acuity (logMAR) without correction (AVsc) and corrected (AVcc) and ORA (Ocular Response Analyzer) parameters: corneal hysteresis $(\mathrm{CH})$, corneal resistance factor (CRF), intraocular pressure calibrated for Goldmann (IOPg) e corneal compensated (IOPcc). Variables had no normal distribution (Kolmogorov-Smirnov test), so that the Wilcoxon signed rank was used for testing the significance on the differences for each variable between morning and afternoon. The Spearman test was used for assessing the correlations between the ORA parameters and refraction, visual acuity in the morning and afternoon, as well as to verify the correlations between the ORA parameters and the variations on refraction and visual acuity. Results: Sphere (E), spherical equivalent (EE), defocus equivalent (ED), AVsc, IOPcc e IOPg varied significantly (Wilcoxon, $\mathrm{p}<0.05$ ) between morning and evening. There was more hyperopia, worse visual acuity, higher pressure and lower $\mathrm{CH}$ in the morning measurements. In the morning measurements, there was a positive correlation (Spearman, $\mathrm{p}<0.05$ ) between EE and ED and IOPcc ( $\mathrm{rs}=0.39$ and 0.34 respectively), but not with IOPg. In the evening measurement, there were no correlations between the refractive and pressure measurements. Negative correlations were observed (Spearman, $\mathrm{p}<0.05)$ between AVsc $(\log \mathrm{MAR})$ and $\mathrm{CH}$ in the morning and in the evening $(\mathrm{rs}=-0.48$ e rs $=-0,51)$, between $\mathrm{E}$ and $\mathrm{CH}$ in the morning and in the evening ( $\mathrm{rs}=-0.66$ and -0.76 ), between $\mathrm{E}$ and $\mathrm{CRF}$ in the morning and in the evening ( $\mathrm{rs}=-0.40$ and -0.47 ), between $\mathrm{EE}$ and $\mathrm{CH}$ in the morning and in the evening ( $\mathrm{rs}=-0.70$ and -0.68 ), between EE and $\mathrm{CRF}$ in the morning and in the evening ( $\mathrm{rs}=-0.41$ and -0.46$)$, between ED and $\mathrm{CH}$ in the morning and in the evening (rs= 0.64 and -0.54$)$ and between ED and CRF in the morning and in the evening ( $\mathrm{rs}=-0.35$ and -0.34 ). There was a significant negative correlation between the morning measurement of CRF and the variation of defocus equivalent $(p=0.05 ; \mathrm{rs}=-0.30)$ and the variation of AVsc ( $\mathrm{p}=0.04$; $\mathrm{rs}=-0.33)$. Conclusion: More hyperopia was recorded in the morning which was associated with higher compensated pressure (IOPcc) but not with IOPg ten years after RK. Lower biomechanical parameters $(\mathrm{CRF}$ e $\mathrm{CH})$ were associated with higher hyperopia and worse visual acuity. A trend was observed for having higher fluctuation on weaker corneas. New studies involving the variables derived from the waveform signals, beyond $\mathrm{CH}$ and $\mathrm{CRF}$ (derived from the applanation pressures) along with data from corneal tomography and wavefront aberrometry are necessary.

Keywords: Keratototmy, radial/adverse effects; Hyperopia; Cornea/physiology; Refractive erros; Visual acuity
\end{abstract}

\section{INTRODUÇÃO}

A ceratotomia radial (CR ou radial keratotomy $-R K$ ) foi introduzida no Brasil em abril de 1981 e, até o advento do excimer laser, correspondeu à técnica cirúrgica mais utilizada para correção da miopia no mundo inteiro. . $^{(1,2)}$ Uma média de 250.000 procedimentos de CR ou RK foram realizadas anualmente nos EUA na primeira metade da década de $90 .{ }^{(3)}$ Atualmente, considerando-se a maior previsibilidade, eficácia e segurança do excimer laser, a RK tornou-se um procedimento histórico. Entretanto, devido ao grande número de pacientes submetidos à RK realizadas no passado, é comum nos depararmos com complicações tardias, destacando-se a hipermetropia. ${ }^{(4,8)}$

A hipermetropia após RK pode resultar de uma hipercorreção imediata ou de um efeito contínuo das incisões ao longo do tempo, o que pode ser exacerbado por um aumento da pressão ocular..$^{(9,10)}$ Também podendo estar relacionada com outras alterações fisiológicas do sistema óptico ocular. ${ }^{(11)}$ De forma geral, o paciente jovem compensa a hipermetropia com a acomodação, havendo evolução gradual para hipermetropia manifesta desde a segunda metade da quarta década de vida. Adicionalmente, a flutuação da acuidade visual e do estado refracional durante o dia comumente agrava o estado de hipermetropia tardia, décadas após a realização da RK. Tal fenômeno é reconhecido e ocorre em até $60 \%$ dos casos desde o pós-operatório inicial, ${ }^{(12,13)}$ podendo persistir em alguns casos. ${ }^{(14,15)} \mathrm{A}$ instabilidade da córnea foi documentada por meio de topografia, cujos índices se relacionaram melhor que a ceratometria central com a flutuação refracional e da acuidade visual não corrigida. ${ }^{(16,17)}$

Há evidências de que a instabilidade da córnea após RK que leva à hipermetropia esteja relacionada com variações da pressão ocular. ${ }^{(17)} \mathrm{Em}$ um estudo experimental, alterações induzidas na pressão ocular foram relacionadas com o grau de aplanamento da córnea em coelhos submetidos à RK... ${ }^{(18)}$ Entretanto, dados conflitantes foram encontrados em outros estudos, que falharam em correlacionar as alterações da pressão ocular ao longo do dia, com as variações refracionais e de acuidade visual encontradas. ${ }^{(15,19,20)}$

Nossa hipótese é que a inconsistência nas associ- 
Tabela 1

\section{Variáveis tabuladas para o estudo}

· ID, idade, sexo, ano da realização da RK, olho, número de incisões

- Grau Esférico (E), Cilindro (C) e Eixo às 9am

- Grau Esférico (E), Cilindro (C) e Eixo às 6pm

- Acuidade Visual sem correção (AVsc) e com correção (AVcc) às $9 \mathrm{am}$

- Acuidade Visual sem correção (AVsc) e com correção (AVcc) às $6 \mathrm{pm}$

- IOPg, IOPcc, CH e CRF às 9am

- IOPg, IOPcc, CH e CRF às $6 \mathrm{pm}$
Tabela 2

Equivalente esférico (EE) e Equivalente desfoco (ED) ${ }^{25}$

- Equivelante Esférico: $\mathrm{EE}=\mathrm{E}+(\mathrm{C} / 2)$

Valor esférico (E) somado a metade do cilindro (C), respeitando-se o sinal

- Equivalente Desfoco: ${ }^{(26)} \mathrm{ED}=[\mathrm{EE}]+[\mathrm{C} / 2]$

Valor absoluto do equivalente esférico (EE) somado a metade do valor absoluto do cilindro

Ex: $+1,00=-2,00 \times 90^{\circ}-\mathrm{EE}=+1+(1 / 2 \times(-2))=+1-1=$ zero (plano) $; \mathrm{ED}=[$ zero $]+(1 / 2 \times[-2])=1$

$-1,00=-2,00 \times 90^{\circ}-\mathrm{EE}=-1+(1 / 2 \times(-2))=-1-1=-2 ; \mathrm{ED}$ $=[-2]+(1 / 2 \times[-2])=3$

Tabela 3

Média e desvio padrão dos parâmetros que variaram significativamente entre 9am e 6pm (Teste de Wilcoxon*)

\begin{tabular}{lcccrr}
\hline Parâmetro & Média - 9am & $\begin{array}{c}\text { Desvio } \\
\text { Padrão - 9am }\end{array}$ & Média - 6pm & $\begin{array}{c}\text { Desvio } \\
\text { Padrão - 6pm }\end{array}$ & valor p* \\
\hline Grau Esférico (D) & 2,49 & 1,73 & 1,76 & 1,72 & $<0,0001$ \\
Equivalente Esférico (D) & 1,59 & 1,49 & 0,89 & 1,57 & $<0,0001$ \\
Equivalente Desfoco (D) & 2,64 & 1,53 & 2,26 & 1,46 & 0,0099 \\
IOPcc (mmHg) & 18,92 & 4,89 & 16,43 & 4,43 & $<0,0001$ \\
IOPg (mmHg) & 15,69 & 1,89 & 14,16 & 4,91 & $<0,0001$ \\
Histerese (mmHg) & 9,29 & 1,89 & 9,78 & 1,52 & 0,0381 \\
AVsc (logMAR) & 0,56 & 0,35 & 0,50 & 0,33 & 0,0205 \\
\hline
\end{tabular}

Tabela 4

Correlações significantes (9am)

\begin{tabular}{|c|c|c|c|}
\hline Equivalente Esférico X CH & & Equivalente Desfoco X CRF & \\
\hline Coeficiente de Spearman (rs)= & -0.7018 & Coeficiente de Spearman $(\mathrm{rs})=$ & 474 \\
\hline Equivalente Esférico $X \stackrel{(\mathrm{p})=}{\mathrm{CRF}}$ & $<0$. & $\begin{array}{r}(\mathrm{p})= \\
\text { Equivalente Esférico } \times \mathbf{C H}\end{array}$ & 25 \\
\hline $\begin{aligned} \text { Coeficiente de Spearman (rs) } & = \\
(\mathrm{p}) & =\end{aligned}$ & $\begin{array}{l}-0.4132 \\
0.0099\end{array}$ & $\begin{array}{r}\text { Coeficiente de Spearman (rs) }= \\
(\mathrm{p})=\end{array}$ & $\begin{array}{l}-0.6613 \\
<0.0001\end{array}$ \\
\hline ente Desfoco X CH & & Equivalente Esféricox CRF & \\
\hline $\begin{aligned} \text { Coeficiente de Spearman (rs) } & = \\
(\mathrm{p}) & =\end{aligned}$ & $\begin{array}{l}-0.6414 \\
<0.0001\end{array}$ & $\begin{aligned} \text { Coeficiente de Spearman }(\mathrm{rs}) & = \\
(\mathrm{p}) & =\end{aligned}$ & $\begin{array}{c}-0.4044 \\
0.0118\end{array}$ \\
\hline
\end{tabular}


ações entre as variações da hipermetropia e da acuidade visual com a pressão ocular seja atribuída a uma imprecisão na medida da pressão ocular com método considerado como o padrão. Alterações das medidas de tonometria de aplanação de Goldmann (TAG) após cirurgias corneanas são bem reconhecidas. ${ }^{(21)}$ Apesar da RK não determinar alteração da paquimetria, o procedimento determina um impacto biomecânico significativo, o que leva a alterações na medida da TAG, podendo inclusive, influenciar ou dificultar o diagnóstico de glaucoma. ${ }^{22,23}$ Novos métodos para medida da pressão ocular foram introduzidos no mercado oftalmológico, destacando-se o ORA (Ocular Response Analyzer, Reichert [Depew, EUA]). O ORA mede a pressão intraocular (intraocular pressure - IOP) calibrada para o padrão Goldmann (IOPg) e calcula uma medida da pressão compensada da córnea (IOPcc) a partir de duas medidas que refletem as propriedades viscoelásticas e biomecânicas da córnea: histerese corneana (corneal hysteresis - $\mathrm{CH}$ ) e o fator de resistência da córnea (corneal resistance factor - CRF). ${ }^{(24)}$ Considerando que o impacto da RK na medida da pressão ocular se dá por alterações nas propriedades biomecânicas da córnea, o estudo destas variáveis é de grande interesse e relevância clínica.

O presente estudo tem o objetivo de verificar variações da refração, da acuidade visual, das medidas da pressão ocular e dos parâmetros biomecânicos da córnea medidos com o ORA pela manhã e à tarde, em pacientes operados de ceratotomia radial, que se apresentaram com interesse em retratamento refrativo. Adicionalmente, foram verificadas as correlações entre os parâmetros biomecânicos da córnea ( $\mathrm{CH}$ e CRF) com a refração, com a acuidade visual e com as variações da refração e da acuidade visual entre manhã e tarde.

\section{Métodos}

O estudo retrospectivo incluiu 38 olhos de 19 pacientes submetidos à RK há mais de 10 anos, que se apresentaram com interesse em retratamento refrativo. $\mathrm{O}$ estudo seguiu os critérios e recomendações da Declaração de Helsinki, e fez parte de um protocolo aprovado pelo comitê de ética para pesquisas em humanos. Todos os pacientes concordaram em participar deste estudo.

Os pacientes foram examinados pela manhã ( 9 am) e à tarde (6 pm), obtendo-se refração esfero-cilíndrica dinâmica (dioptrias [D]), acuidade visual ( $\log$ MAR) sem correção (AVsc), acuidade visual corrigida (AVcc) e os parâmetros do ORA (mmHg). Exame oftalmológico completo foi realizado em todos os casos. Os dados relevantes ao estudo (Tabela 1) foram tabulados no Excell 97-2003 (Microsoft, EUA).

$\mathrm{O}$ equivalente esférico (EE) e o equivalente desfoco (ED) foram calculados em dioptrias (D), de acordo com as fórmulas (Tabela 2). Estudos estatísticos foram realizados utilizando-se o BioEstat 5,0 (download gratuito - Instituto Mamirauá, Brasil).

Considerando a não distribuição normal das variáveis (teste de Kolmogorov-Smirnov), o teste de Wilcoxon rank sum foi utilizado para verificar diferenças entre as medidas da manhã e as da tarde de cada variável. As médias e os desvios padrão foram calculados para cada variável pela manhã e à tarde.

As diferenças entre as medidas da manhã e as da tarde do grau esférico (E), do cilindro $(\mathrm{C})$, do equivalente esférico (EE), do equivalente desfoco (ED) e da acuidade visual corrigida ( $\mathrm{AV} c \mathrm{c})$ e não corrigida $(\mathrm{AVsc})$ foram calculadas.

Considerando a não distribuição normal das variáveis (teste de Kolmogorov-Smirnov), o teste de Spearman foi utilizado para verificar correlações das medidas do ORA pela manhã (CH, CRF, IOPg e IOPcc) com o estado refracional (E, C, EE e ED) e com as medidas de acuidade visual (AVsc e AVcc) pela manhã. As mesmas correlações foram calculadas para as medidas à tarde.

O teste de Spearman foi também utilizado para verificar correlações entre as medidas do ORA (IOPcc, $\mathrm{IOPg}, \mathrm{CH}$ e CRF) pela manhã e à tarde e as variações da refração e da acuidade visual. Significância estatística foi considerada para valores p menores que 0,05 .

\section{Resultados}

Observou-se diferença estatisticamente significante no grau esférico (E), equivalente esférico (EE), equivalente desfoco (ED), AVsc, IOPcc e IOPg entre manhã e tarde (Wilcoxon, $\mathrm{p}<0,05$ [Tabela 3]).

Vinte e nove dos 38 olhos (76,3\%) apresentaram uma diferença do grau esférico pela manhã e a tarde maior que $0,50 \mathrm{D}$; em 12 olhos (38\%) esta diferença foi superior a 1D. A pressão compensada (IOPcc) foi maior pela manhã em 33 olhos (87\%) e a IOPg, maior pela manhã em 30 olhos (79\%). A visão não corrigida foi melhor à tarde em 29 olhos (73\%). Apenas dois olhos apresentaram maior hipermetropia na medida da tarde, sendo que em um deles a pressão ocular foi maior à tarde e em outro a pressão não variou.

O grau esférico (E) e o equivalente esférico (EE) apresentaram uma redução da hipermetropia durante o 
Eq. Esférico x IOPcc - 9am

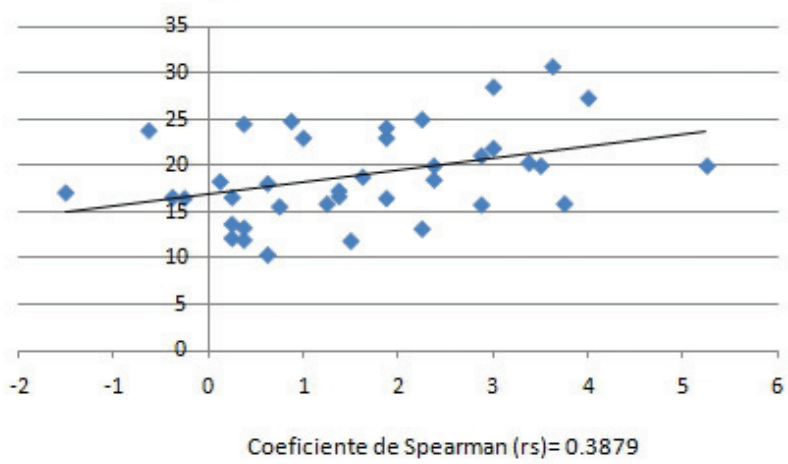

$(p)=0.0161$
Eq. Desfoco $\times 10 P c c-9 a m$

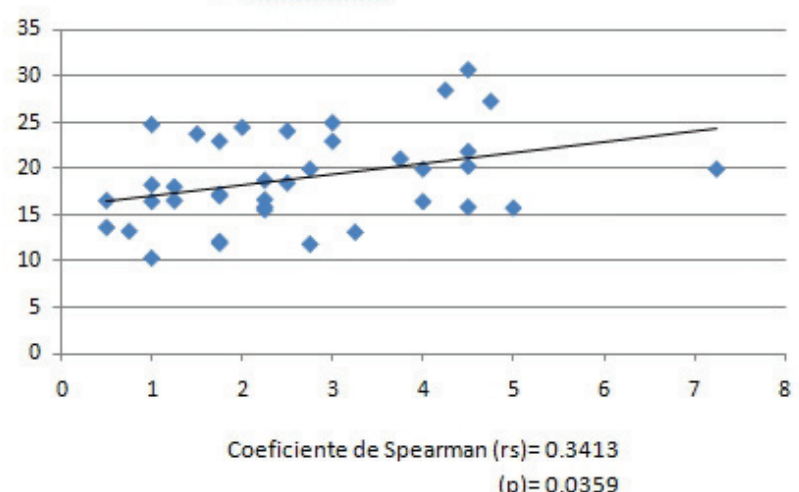

Figura 1: Correlações entre IOPcc e equivalente esférico (EE) e equivalente desfoco (ED)

Eq. Esférico x Histerese, 9am

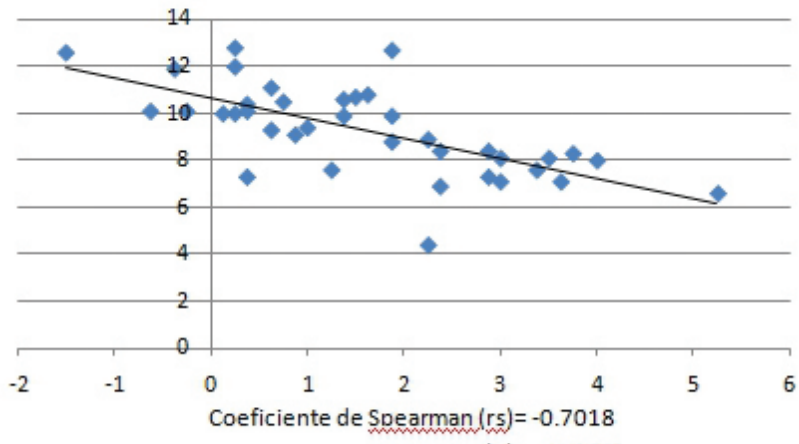

$(p)=<0.0001$

Eq. Desfoco x Histerese, 9am

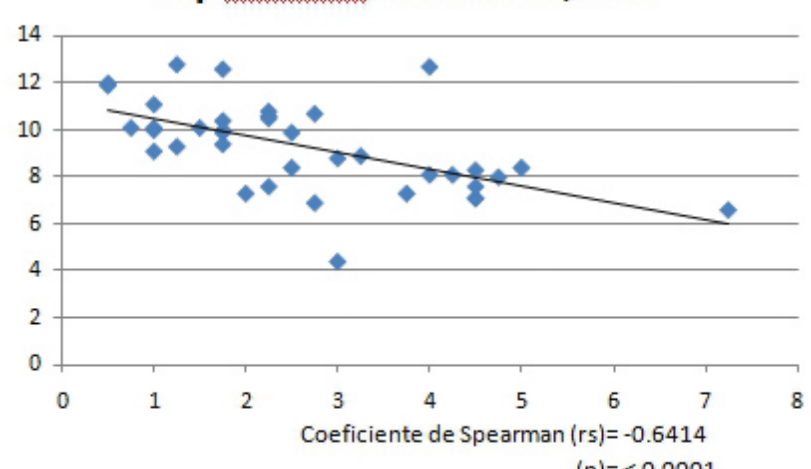

$(p)=<0.0001$
Eq. Esférico x CRF, 9am

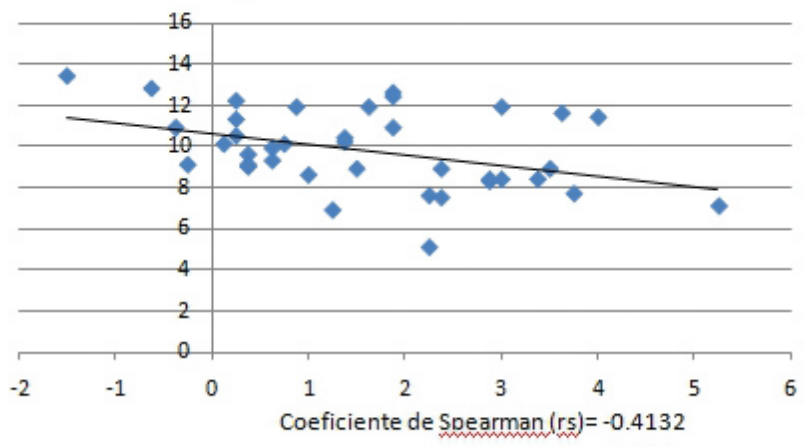

$(p)=0.0099$

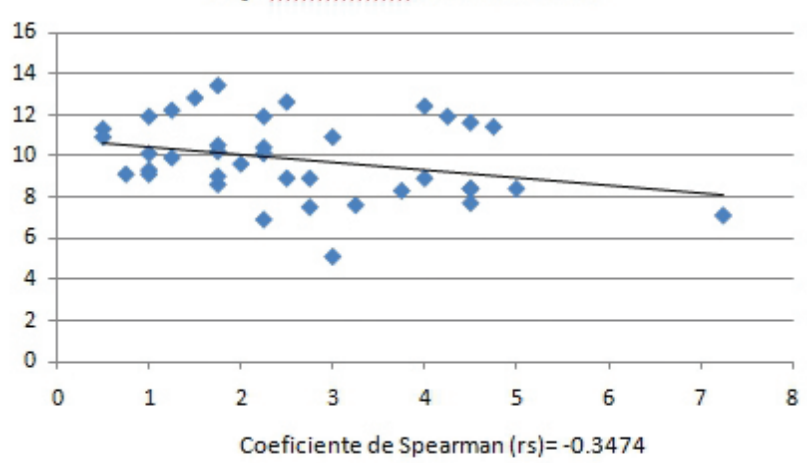

$(p)=0.0325$

Figura 2: Correlações significativas entre equivalente esférico e equivalente desfoco e CH e CRF pela manhã 
Grau Esférico (E) x CH, 9am

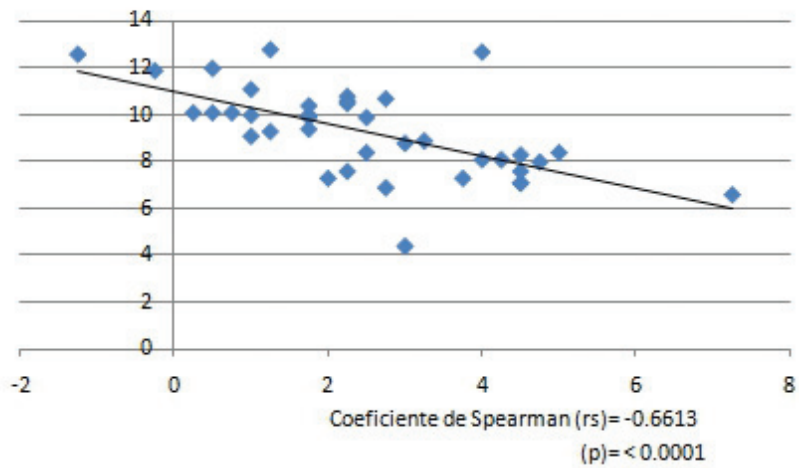

Grau Esférico (E) x CH, 6pm

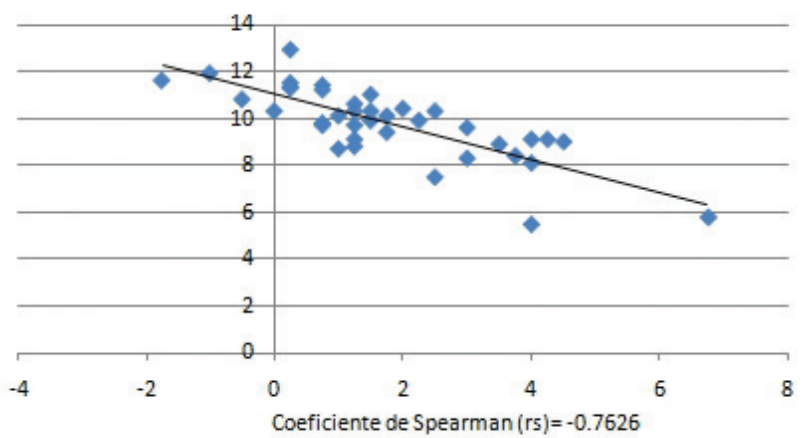

$(p)=<0.0001$

Figura 3: Correlações entre grau esférico $(\mathrm{E})$ e histerese $(\mathrm{CH})$ às 9am e 6pm

Delta Equivalente Desfoco x CRF 9am

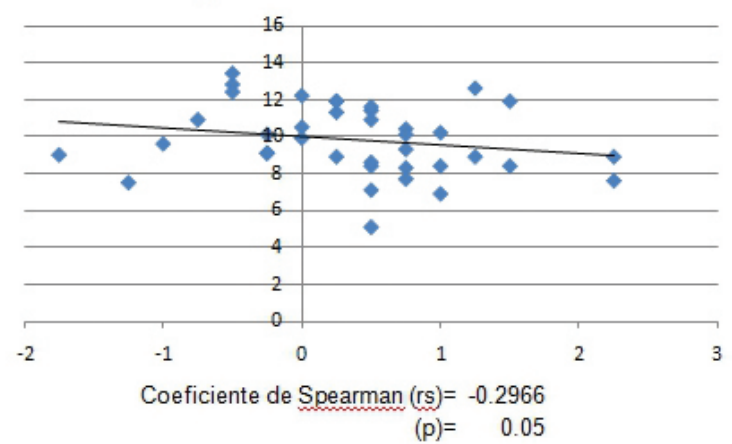

Figura 4: Correlação entre a diferença do equivalente desfoco e o CRF 9am

dia ( $<<0,0001)$. O grau esférico apresentou redução de $2,49 \pm 1,73$ para $1,59 \pm 1,72$. O EE apresentou redução de $1,59 \pm 1,49$ para $0,89 \pm 1,57$. O equivalente desfoco reduziu de $2,64 \pm 1,53$ para $2,26 \pm 1,46(\mathrm{p}=0,0099)$. A média da AVsc melhorou ao longo do dia, de 0,56 $\pm 0,35$ (20/73) para $0,50 \pm 0,33(20 / 63)$, o que foi estatisticamente significante ( $\mathrm{p}=0,0205$, Tabela 3$)$.

$\mathrm{O}$ valor do cilindro $(\mathrm{C})$ não variou de forma significativa $(\mathrm{p}=0,49)$, bem como o CRF $(\mathrm{p}=0,57)$ e a AVcc $(\mathrm{p}=0,26)$. A média da acuidade visual corrigida passou de $0,3 \pm 0,29(20 / 40)$ para $0,63 \pm 0,23(20 / 29)$, mas tal variação não teve significância estatística $(\mathrm{p}=0,26)$.

Nas medidas pela manhã, observaram-se correlações positivas (Spearman, $\mathrm{p}<0,05$ ) do EE e do ED com IOPcc (rs=0,39 e 0,34 respectivamente [Figura 1]), mas
Delta AVsc (LogMar) x CRF 9am

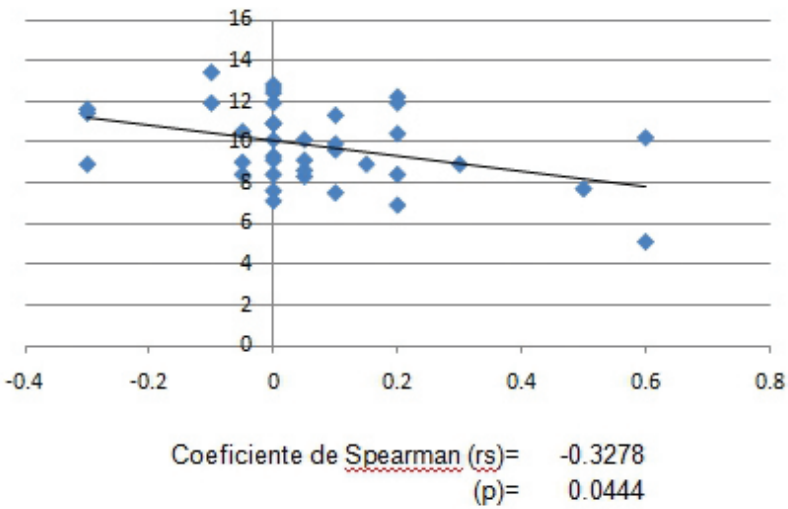

Figura 5: Correlação entre a diferença da AVsc e o CRF 9am

não com IOPg. Nas medidas da tarde, não houve correlações significantes entre os parâmetros refracionais e os pressóricos.

Observaram-se correlações negativas (Spearman, $p<0,05)$ entre o grau esférico (E), equivalente esférico (EE) e equivalente desfoco (ED) e os parâmetros biomecânicos $\mathrm{CH}$ e CRF pela manhã (Tabela 4, Figura 2) e à tarde. As maiores correlações foram encontradas entre o grau esférico (E) e $\mathrm{CH}$ pela manhã e a tarde (rs= $-0,66$ e -0,76, Figura 3). Foram encontradas correlações significantes e negativas entre $\mathrm{E}$ e CRF pela manhã e à tarde (rs= - 0,40 e -0,47), entre $\mathrm{EE}$ e $\mathrm{CH}$ pela manhã e à tarde (rs= $-0,70$ e $-0,68)$, entre EE e CRF pela manhã e à tarde (rs=-0,41 e - 0,46 ), entre ED e CH pela manhã e a tarde (rs= - 0,64 e -0,54) e entre ED e CRF pela manhã e 
a tarde (rs $=-0,35$ e $-0,34)$.

Observou-se correlação significativa entre a diferença do equivalente desfoco entre manhã e tarde e o CRF medido às 9am (Figura 4).

Houve também correlação significativa entre a diferença da acuidade visual sem correção e o CRF medido às 9am (Figura 5). Uma maior variação de mais desfoco e pior AVsc pela manhã foi relacionada com valores de CRF mais baixos.

\section{Dıscussão}

A hipermetropia secundária, que ocorre décadas após RK, é uma complicação relativamente comum ${ }^{(4,6,27-}$ ${ }^{29)}$, que pode ser agravada por instabilidade da córnea e flutuação refracional durante o dia. Neste estudo, observamos uma variação significativa da refração e da acuidade visual entre as medidas da manhã e da tarde, que foi relacionada com propriedades biomecânicas mais baixas da córnea e com maior pressão compensada (IOPcc) em pacientes operados de ceratotomia radial.

Ocorre um aumento da incidência e da magnitude da hipermetropia ao longo do período pós-operatório de RK. O Prospective Evaluation of Radial Keratotomy Study (PERK) foi um estudo prospectivo, multicêntrico, composto de nove centros de investigação, que determinou o início de uma era científica para a Cirurgia Refrativa. ${ }^{(5)}$ No estudo PERK, todas as cirurgias foram realizadas com oito incisões, variando-se apenas a profundidade das incisões e a zona óptica (ZO) livre, de acordo com a espessura corneana e a refração do paciente. Os resultados do PERK demostram a tendência de hipermetropização, com $43 \%$ dos casos apresentando em 10 anos, um aumento de efeito superior a +1.00 dioptria. ${ }^{(5)}$ Um determinado grau de hipermetropização é esperado com o envelhecimento, ${ }^{(30)}$ uma vez que há perda progressiva da acomodação.

Denomina-se hipermetropia latente o grau compensado pela acomodação e aparece na refração sobre cicloplegia. Com a perda progressiva da acomodação esse grau deixa de ser latente e torna-se facultativo e posteriormente manifesto. Entretanto, no estudo do PERK, além dos olhos submetidos à RK, os olhos contralaterais que não foram submetidos à cirurgia, também foram avaliados e nenhum destes olhos evoluiu com uma alteração de grau acima de 1.00 dioptria em direção à hipermetropia.

Adicionalmente, Deitz et al. ${ }^{(4)}$ relataram $54 \%$ de hipermetropia com 8 anos de seguimento. Guedes et al. ${ }^{(6)}$ reportaram uma incidência de $42 \%$ de hipermetropia que variou entre $+1.00 \mathrm{a}+5.75$, mais de 10 anos após RK. Tais achados tardios contrastam com o pós-operatório de um ano em que apenas $4,2 \%$ dos olhos apresentavam-se hipermétropes ${ }^{(6)}$.

Entre os fatores de risco mais importantes para o processo de hipermetropização tardio após RK, encontra-se ZO menores, maior número de incisões, maior profundidade das incisões, a presença de incisões transversas e menor estroma residual posterior livre da incisão. ${ }^{(4,6)}$ Todas estas características da cirurgia estão relacionadas com um maior trauma nas fibras de colágeno da córnea, o que provoca maior efeito da cirurgia e maior indução de aplanamento e possivelmente de irregularidade. ${ }^{(31)} \mathrm{Com}$ isso, quanto maior o impacto biomecânico causado pela cirurgia, maior o aplanamento. Demonstrou-se que a prevenção da hipermetropia é possível se for mantida uma zona periférica livre de incisões. ${ }^{(32)}$ Estudos realizados por Meek et al. ${ }^{(33,35)}$ demonstram que maior resistência biomecânica é conferida às lamelas periféricas da córnea, que apresentam maior reticulação (crosslinking), capacidade natural do colágeno.

Neste estudo, parâmetros biomecânicos (CRF e $\mathrm{CH})$ mais baixos foram associados com maior hipermetropia e pior acuidade visual tanto nas medidas pela manhã, como à tarde. Tal fato pode ser relacionado com uma cirurgia que determinou maior alteração e enfraquecimento da córnea, mas também poderia ser explicado por tratar-se de uma córnea "mais fraca" desde o pré-operatório. Resultados insatisfatórios de RK com severa indução de irregularidade e piora da acuidade visual foram reportados em casos de ceratocone. ${ }^{(38,37)}$ Entretanto, nenhum estudo foi capaz de verificar se há correlação independente da espessura pré-operatória com a hipermetropia após RK. Adicionalmente, não eram disponíveis metodologias mais sofisticadas para estudo tomográfico ${ }^{(38,39)}$ ou biomecânico $^{(24,40)}$ da córnea no pré-operatorio de RK. De qualquer forma, sendo o estado biomecânico mais fraco causado pela RK ou a razão da hipercorreção da $\mathrm{RK}$, o presente estudo identifica diversas correlações negativas entre o grau esférico (E), equivalente esférico (EE) e equivalente desfoco (ED) e os parâmetros biomecânicos $\mathrm{CH}$ e CRF. Ou seja, quanto maior o grau de hipermetropia, menores o CH e CRF. Com isso, contundentemente podemos afirmar que, para o momento atual, existe uma associação entre o estado biomecânico mais fraco com o grau de hipermetropia.

Observou-se nas medidas pela manhã, correlações positivas entre o estado refracional (EE e ED) e a 
pressão compensada da córnea (IOPcc). Tal observação corrobora com o conceito de que a instabilidade da córnea após RK esteja relacionada com variações da pressão ocular. ${ }^{(17)}$ É importante notar que não houve correlações com a IOPg. Tais achados suportam nossa hipótese de que a inconsistência nas associações entre as variações da hipermetropia e da pressão ocular, observada em alguns estudos, ${ }^{(15)}$ está relacionada com alterações das medidas de tonometria de aplanação de Goldmann (TAG) após RK. A IOPcc é uma medida que considera a resposta viscoelástica da córnea para gerar um valor pressórico menos influenciado pela estrutura corneana, ${ }^{(24)}$ o que reduz o impacto das alterações da córnea após cirurgias nesta medida da pressão. ${ }^{(41)}$ Nas medidas da tarde, não houve correlações significantes entre os parâmetros refracionais e os pressóricos, o que pode ser explicada pela redução significativa encontrada da pressão ocular ao longo do dia, o que reduz a influência da pressão no estado refracional.

Não se observou correlação entre a flutuação (diferenças entre manhã e tarde) no grau esférico e no equivalente esférico e $\mathrm{CH}$ e CRF. Entretanto, houve associação entre a variação do equivalente desfoco e o CRF medido pela manhã. Esta tendência de haver maior flutuação relacionada com córneas mais fracas biomecanicamente, merece novos estudos envolvendo parâmetros biomecânicos mais avançados, como os derivados do sinal do ORA. Existem 38 novas variáveis derivadas do sinal de reflexo da córnea durante o exame do ORA*, além dos parâmetros $\mathrm{CH}$ e CRF, que são derivados das pressões de aplanamento do ORA. Adicionalmente, variáveis topográficas, tomográficas da córnea e de aberrometria total devem ser estudadas.

A flutuação refracional juntamente com o estado biomecânico da córnea e a pressão ocular devem ser considerados individualmente em pacientes submetidos à RK, com interesse em retratamento refrativo. Tais variáveis são também importantes no caso de se considerar cirurgia de catarata. ${ }^{(42-44)} \mathrm{O}$ aplanamento da córnea pode ser acentuado no pós-operatório imediato, levando a uma relativa surpresa hipermetrópica transitória. Tal fenômeno ocorre devido ao edema corneano juntamente com a possível hipertensão ocular. O maior conhecimento sobre as variáveis biomecânicas e do impacto da pressão ocular sobre a flutuação da córnea abre definitivamente um novo horizonte para entender os achados clínicos e personalizar tratamentos neste complexo grupo de pacientes.

\section{RefERÊNCiaS}

1. Radial keratotomy for myopia. American Academy of Ophthalmology. Ophthalmology. 1993;100(7):1103-15.

2. Rowsey JJ, Morley WA. Surgical correction of moderate myopia: which method should you choose? I. Radial keratotomy will always have a place. Surv Ophthalmol. 1998;43(2):147-56.

3. Leaming DV. Practice styles and preferences of ASCRS members-1993 survey. J Cataract Refract Surg. 1994;20(4):459-67.

4. Deitz MR, Sanders DR, Raanan MG. Progressive hyperopia in radial keratotomy. Long-term follow-up of diamond-knife and metal-blade series. Ophthalmology. 1986;93(10):1284-9.

5. Waring GO 3rd, Lynn MJ, McDonnell PJ. Results of the prospective evaluation of radial keratotomy (PERK) study 10 years after surgery. Arch Ophthalmol. 1994;112(10):1298-308.

6. Guedes J, Andrade C, Andrade M, Guedes A, Ambrósio Júnior R. Hipermetropia pós-cerototomia radial. Rev Bras Oftalmol. 2003;62(10):700-5.

7. John ME. High hyperopia after radial keratotomy. J Cataract Refract Surg. 1993;19(3):446-7. Comment on J Cataract Refract Surg. 1993;19(1):88-9.

8. Kirkconnell WS. Progressive hyperopia following radial keratotomy. J Cataract Refract Surg. 1989;15(6):719.

9. Lindquist TD, Williams PA, Lindstrom RL. Surgical treatment of overcorrection following radial keratotomy: evaluation of clinical effectiveness. Ophthalmic Surg. 1991;22(1):12-5.

10. Pulaski JP. Overcorrections with radial keratotomy. J Cataract Refract Surg. 1996;22(6):653-4. Comment on J Cataract Refract Surg. 1996;22(1):51-8.

11. Werblin TP, Phillips S, Krider D. Progressive hyperopia secondary to RK or just normal physiology? J Cataract Refract Surg. 2007;33(7):1343-4.

12. Bores LD, Myers W, Cowden J. Radial keratotomy: an analysis of the American experience. Ann Ophthalmol. 1981;13(8):941-8.

13. Hoffer KJ, Darin JJ, Pettit TH, Hofbauer JD, Elander R, Levenson JE. UCLA clinical trial of radial keratotomy. Preliminary report. Ophthalmology. 1981;88(8):729-36.

14. McDonnell PJ, Nizam A, Lynn MJ, Waring GO 3rd. Morningto-evening change in refraction, corneal curvature, and visual acuity 11 years after radial keratotomy in the prospective evaluation of radial keratotomy study. The PERK Study Group. Ophthalmology. 1996;103(2):233-9.

15. Schanzlin DJ, Santos VR, Waring GO 3rd, Lynn M, Bourque L, Cantillo N, et al. Diurnal change in refraction, corneal curvature, visual acuity, and intraocular pressure after radial keratotomy in the PERK Study. Ophthalmology. 1986;93(2):167-75.

16. McDonnell PJ, McClusky DJ, Garbus JJ. Corneal topography and fluctuating visual acuity after radial keratotomy. Ophthalmology. 1989;96(5):665-70.

17. Kemp JR, Martinez CE, Klyce SD, Coorpender SJ, McDonald $\mathrm{MB}$, Lucci L, et al. Diurnal fluctuations in corneal topography 10 years after radial keratotomy in the Prospective Evaluation of Radial Keratotomy Study. J Cataract Refract Surg. 1999;25(7):904-10.

18. Busin M, Yau CW, Avni I, McDonald MB, Kaufman HE. The effect of changes in intraocular pressure on corneal curvature after radial keratotomy in the rabbit eye. Ophthalmology. 1986;93(3):331-4.

19. Kwitko S, Garbus JJ, Hwang DG, Gauderman WJ, McDonnell PJ. Computer-assisted study of diurnal variation in corneal topography after penetrating keratoplasty. Ophthalmic Surg. 1992;23(1):10-6. 
20. Santos VR, Waring GO 3rd, Lynn MJ, Schanzlin DJ, Cantillo $\mathrm{N}$, Espinal ME, et al. Morning-to-evening change in refraction, corneal curvature, and visual acuity 2 to 4 years after radial keratotomy in the PERK Study. Ophthalmology. 1988;95(11):1487-93.

21. Chihara E. Assessment of true intraocular pressure: the gap between theory and practical data. Surv Ophthalmol. 2008;53(3):203-18.

22. Faucher A, Grégoire J, Blondeau P. Accuracy of Goldmann tonometry after refractive surgery. J Cataract Refract Surg. 1997;23(6):832-8.

23. Scheuerle AF, Martin M, Voelcker HE, Auffarth G. Undetected development of glaucoma after radial keratotomy. J Refract Surg. 2008;24(1):51-4.

24. Luce DA. Determining in vivo biomechanical properties of the cornea with an ocular response analyzer. J Cataract Refract Surg. 2005;31(1):156-62.

25. Waring GO 3rd. Standard graphs for reporting refractive surgery. J Refract Surg. 2000;16(4):459-66. Review. Erratum in: J Refract Surg. 2001;17(3):following table of contents.

26. Holladay JT, Lynn MJ, Waring GO 3rd, Gemmill M, Keehn GC, Fielding B. The relationship of visual acuity, refractive error, and pupil size after radial keratotomy. Arch Ophthalmol. 1991;109(1):70-6.

27. Hoffer KJ, Darin JJ, Pettit TH, Hofbauer JD, Elander R, Levenson JE. Three years experience with radial keratotomy. The UCLA study. Ophthalmology. 1983;90(6):627-36.

28. Binder PS. Four-year postoperative evaluation of radial keratotomy. Arch Ophthalmol. 1985;103(6):779-80.

29. Bodoia RD. Progressive hyperopia in radial keratotomy. Ophthalmology. 1987;94(7):895-6.

30. Wang Q, Klein BE, Klein R, Moss SE. Refractive status in the Beaver Dam Eye Study. Invest Ophthalmol Vis Sci. 1994;35(13):4344-7.

31. Olson RJ, Biddulph MC. Hyperopia, anisometropia, and irregular astigmatism in a patient following revisional radial keratotomy. Ophthalmic Surg. 1992;23(11):782-3.

32. Krumeich JH, Daniel J, Gast R. The suction bridge for radial keratotomy may avoid late hyperopic shift. J Refract Surg. 1997;13(4):367-73.

33. Kamma-Lorger CS, Boote C, Hayes S, Moger J, Burghammer M, Knupp C, et al. Collagen and mature elastic fibre organisation as a function of depth in the human cornea and limbus. J Struct Biol. 2010;169(3):424-30.

34. Abahussin M, Hayes S, Knox Cartwright NE, Kamma-Lorger CS, Khan Y, Marshall J, Meek KM. 3D collagen orientation study of the human cornea using X-ray diffraction and femtosecond laser technology. Invest Ophthalmol Vis Sci. 2009;50(11):5159-64.
35. Boote C, Hayes S, Jones S, Quantock AJ, Hocking PM, Inglehearn $\mathrm{CF}$, et al. Collagen organization in the chicken cornea and structural alterations in the retinopathy, globe enlarged (rge) phenotype-an X-ray diffraction study. J Struct Biol. 2008;161(1):1-8.

36. Durand L, Monnot JP, Burillon C, Assi A. Complications of radial keratotomy: eyes with keratoconus and late wound dehiscence. Refract Corneal Surg. 1992;8(4):311-4.

37. Ellis W. Radial keratotomy in a patient with keratoconus. J Cataract Refract Surg. 1992;18(4):406-9.

38. Ambrósio R Jr, Alonso RS, Luz A, Coca Velarde LG. Cornealthickness spatial profile and corneal-volume distribution: tomographic indices to detect keratoconus. J Cataract Refract Surg. 2006;32(11):1851-9.

39. Luz A, Ursulio M, Castañeda D, Ambrosio Júnior R. Progressão da espessura corneana do ponto mais fino em direção ao limbo: estudo de uma população normal e de portadores de ceratocone para criação de valores de referência. Arq Bras Oftalmol. 2006;69(4):579-83.

40. Fontes BM, Ambrósio R Jr, Alonso RS, Jardim D, Velarde GC, Nosé W. Corneal biomechanical metrics in eyes with refraction of -19.00 to $+9.00 \mathrm{D}$ in healthy Brazilian patients. J Refract Surg. 2008;24(9):941-5.

41. Pepose JS, Feigenbaum SK, Qazi MA, Sanderson JP, Roberts CJ. Changes in corneal biomechanics and intraocular pressure following LASIK using static, dynamic, and noncontact tonometry. Am J Ophthalmol. 2007;143(1):39-47.

42. Osher RH. Transient pseudophakic hyperopia after previous radial keratotomy. J Cataract Refract Surg. 2009;35(12):2176; author reply 2176. Comment on J Cataract Refract Surg. 2009;35(4):759-65.

43. Koch DD, Liu JF, Hyde LL, Rock RL, Emery JM. Refractive complications of cataract surgery after radial keratotomy. Am J Ophthalmol. 1989;108(6):676-82.

44. Centurion V, Caballero JC. Hyperopic shift after phacoemulsification in eyes with previous radial keratotomy [Video]. J Cataract Refract Surg. 200521(3).
Autor para Correspondência:
Dr. Renato Ambrósio Jr
Rua Conde de Bonfim, 211/712
CEP 20520-050 - Tijuca - Rio de Janeiro (RJ), Brasil
Telefone: (21) 2234-4233; (21) 2274- 5694;
(21) 7892-2131
e-mail: renatoambrosiojr@terra.com.br 